\title{
Radiative lepton mass and muon $g-2$ with suppressed lepton flavor and CP violations
}

\author{
Wen Yin \\ Department of Physics, Tohoku University, \\ Sendai, Miyagi 980-8578, Japan \\ Department of Physics, Faculty of Science, The University of Tokyo, \\ Bunkyo-ku, Tokyo 113-0033, Japan \\ E-mail: yin.wen.b3@tohoku.ac.jp
}

ABSTRACT: The recent experimental status, including the confirmation of the muon $g-2$ anomaly at Fermilab, indicates a Beyond Standard Model (BSM) satisfying the following properties: 1) it enhances the $g-22$ ) suppresses flavor violations, such as $\mu \rightarrow e \gamma, 3$ ) suppresses CP violations, such as the electron electric dipole moment (EDM). In this letter, I show that the eigenbasis of the mass matrix and higher dimensional photon operators can be automatically aligned if the masses of heavy leptons are generated radiatively together with the $g-2$. As a result, the muon $g-2$ is enhanced but the EDM of the electron and $\mu \rightarrow e \gamma$ rate are naturally suppressed. Phenomenology and applications of the mechanism to the B-physics anomalies are argued.

Keywords: Beyond Standard Model, CP violation, Cosmology of Theories beyond the SM, Supersymmetric Standard Model

ARXIV EPRINT: 2103.14234 


\section{Contents}

$\begin{array}{llr}1 & \text { Introduction } & 1\end{array}$

2 Heavy lepton from radiative correction. 2

$\begin{array}{llr}3 & \text { Lepton-slepton-bino-like system } & 6\end{array}$

4 Discussion and conclusions $\quad 8$

A RG analysis of the wave function renormalization $\quad 9$

\section{Introduction}

The long-standing discrepancy of the anomalous muon magnetic moment $(g-2)$ observed at the Brookhaven National Laboratory [1] has been recently confirmed by Fermilab [2, 3]. The combined $g-2$ anomaly is

$$
\Delta a_{\mu}=a_{\mu}^{\mathrm{EXP}}-a_{\mu}^{\mathrm{SM}}=(25.1 \pm 5.9) \times 10^{-10},
$$

where $a_{\mu}^{\mathrm{SM}}$ is the standard model $(\mathrm{SM})$ prediction of the $(g-2) / 2$ (See also refs. [410]). ${ }^{1} a_{\mu}^{\mathrm{EXP}}$ is the combined updated experimental value (See also refs. [1, 4, 13-15].) The deviation is around $4.2 \sigma$. (If we adopt the R-ratio analysis in [7] it is at $4.5 \sigma$ level.) There are also anomalies relevant to B-physics, especially the lepton non-universality [16, 17]. For concreteness, we mainly focus on the $g-2$, while our mechanism can also apply to models for explaining the lepton non-universality as will be discussed in the last section.

The $g-2$ result strongly implies beyond SM (BSM) particle(s) coupled to the SM leptons, especially the muon. The result, together with the non-observation of the charged lepton EDM and the charged lepton flavor violation, provides a hint to the flavor and CP structure of the BSM. On the other hand, in collider experiments, there is no evidence of the direct productions of the BSM particles, which may suggest the BSM particles are heavy. If the BSM particles are heavier than $\mathrm{TeV}$, the sizable muon $g-2$ should be generated via a process without a chirality suppression. This implies that a lepton Yukawa coupling obtain a huge loop contribution from any diagram for the $g-2$ by removing the photon external line. Therefore, in this Letter, I would like to study the radiative generation of the lepton Yukawa couplings in the context of the muon $g-2$. See e.g. refs. [18-21] for the SUSY and other theory relating the lepton $g-2$ and the fermion mass hierarchy.

\footnotetext{
${ }^{1}$ See also ref. [7] for the lattice result of the muon $g-2$, which is smaller than that from the $R$-ratio approach. The explanation of the $g-2$ within the SM is an important topic but we may need further checks $[11,12]$.
} 

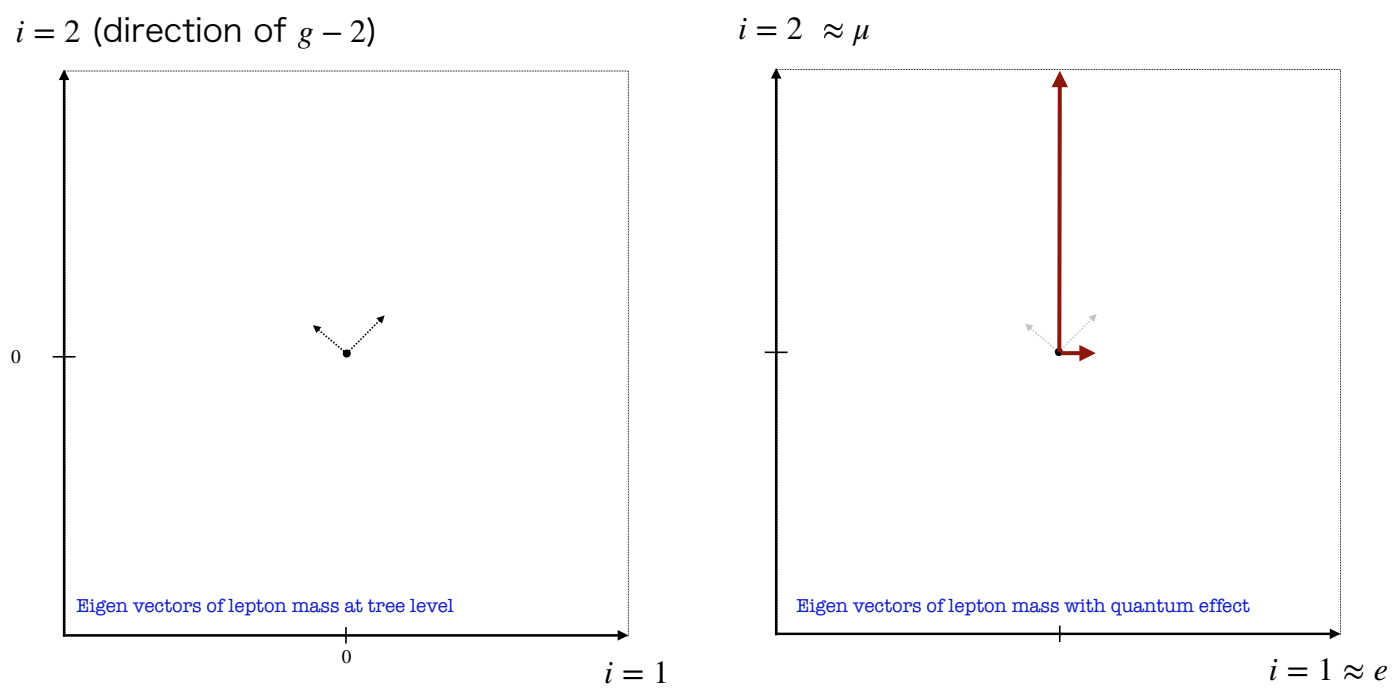

Figure 1. The idea of the mass-interaction alignment mechanism is shown. We take the treelevel mass eigenvectors towards arbitrary directions in the flavor space (left panel). If the only one radiative induced mass, relevant to the $g-2$ contribution, is dominant, the mass vector of the heavier lepton (the vertical red allow) is automatically aligned with the interaction vector of the " $g-2$ " (along the y-axis) in flavor space as shown in the left panel. If the heavier mass vector is the muon and the lighter is the electron, the $\mu \rightarrow e \gamma$ and the electron EDM are highly suppressed. The muon EDM may be generated which is a prediction of this mechanism.

In particular, I show that the $\mu \rightarrow e \gamma$ rate and the electron EDM are naturally suppressed when the muon $g-2$ is explained together with inducing the radiative lepton mass. This is because the induced higher dimensional photon coupling is automatically aligned with the eigenstate vector of the muon mass in the flavor space (See figure 1). We also discuss the possible UV physics for enough suppressions to evade the experimental bounds in the appendix.

\section{$2 \quad$ Heavy lepton from radiative correction.}

Let me give a general discussion to present the idea. For simplicity let us consider leptons with two flavors. Later they will be identified as electron and muon. I will also assume that the BSM particles couple to only one combination of the leptons without flavor/CP symmetry. As an example of this type of model, we can consider a Lagrangian involving the SM leptons,

$$
\mathcal{L}_{\text {int }} \supset-\sum_{\kappa_{1} \kappa_{2}=1,2} \hat{y}_{\kappa_{1}, \kappa_{2}} H_{0} \overline{\hat{L}}_{\kappa_{1}} \hat{e}_{\kappa_{2}}-\sum_{\kappa=1,2}\left(c_{\kappa}^{L} O^{\mathrm{BSM}, L} \hat{L}_{\kappa}+c_{\kappa}^{R} O^{\mathrm{BSM}, R} \hat{e}_{\kappa}\right)
$$

where $\hat{L}_{\kappa}\left(\hat{e}_{\kappa}\right)$ are the left (right) handed charged leptons in a generic basis, $O^{\mathrm{BSM}, L / R}$ (composite) operators of BSM fields and $c^{L / R}$ the coupling to the BSM operators. Our "single family" assumption of the BSM coupling appears from that $O^{\mathrm{BSM}, L / R}$ do not have the flavor index $\kappa$, i.e. there are no copies of $O^{\mathrm{BSM}, L / R}$. Note that we did not assume any flavor structure to get eq. (2.1), and thus this is a generic form. The absence of the 
flavor index in $O^{\mathrm{BSM}, L / R}$ can be accidentally realized even if the BSM fields are many (see the model in the next section). From this Lagrangian we can redefine the lepton flavor so that $L_{2} \equiv \sin \theta_{L} \hat{L}_{1}-\left(\cos \theta_{L}\right)^{*} \hat{L}_{2}, e_{2} \equiv \sin \theta_{R} \hat{e}_{1}-\left(\cos \theta_{R}\right)^{*} \hat{e}_{2}$. where $\left(\cos \theta_{L}\right)^{*} \equiv$ $-c_{2}^{L} / \sqrt{\sum_{\kappa}\left|c_{\kappa}^{L}\right|^{2}}, \sin \theta_{L} \equiv c_{1}^{L} / \sqrt{\sum_{\kappa}\left|c_{\kappa}^{L}\right|^{2}}$, represent the rotation of the basis. We get the definition of $\cos \theta_{R}, \sin \theta_{R}$ with $L$ replaced to $R$ everywhere. $L_{1}$ and $e_{1}$ can be defined by $L_{1} \equiv \cos \theta_{L} \hat{L}_{1}+\left(\sin \theta_{L}\right)^{*} \hat{L}_{2}, e_{1} \equiv \cos \theta_{R} \hat{e}_{1}+\left(\sin \theta_{R}\right)^{*} \hat{e}_{2}$. Then we get the Lagrangian in the BSM interaction basis,

$$
\mathcal{L}_{\text {int }} \supset-\sum_{i j} y_{i j} H_{0} \bar{L}_{i} e_{j}-\left(\sqrt{\sum_{\kappa}\left|c_{\kappa}^{L}\right|^{2}} O^{\mathrm{BSM}, L} L_{2}+\sqrt{\sum_{\kappa}\left|c_{\kappa}^{R}\right|^{2}} O^{\mathrm{BSM}, R} e_{2}\right)
$$

Here $y_{i j}$ is a function of $\theta_{L}, \theta_{R}$ and $\hat{y} . y_{i j}$ has non-vanishing off-diagonal components in general. If each component of $\hat{y}_{\kappa_{1}, \kappa_{2}}$ is same order, $\theta_{L, R}=\mathcal{O}(1)$ and there is no cancelations, each component of $y_{i j}$ is the same order.

To be more generic, we consider the interacting Lagrangian at the cutoff scale, $\mu \sim$ $\Lambda_{\text {cutoff }}$, with normalized kinetic terms given as

$$
\mathcal{L}_{\text {int }} \supset-\sum_{i j} y_{i j} H_{0} \bar{L}_{i} e_{j}-\mathcal{L}_{\mathrm{BSM}}\left[L_{2}, e_{2}, \mathcal{O}\right]
$$

where $L_{i}\left(e_{j}\right)$ are the left (right) handed charged leptons in the BSM interaction basis, $H_{0}=v+\frac{h}{\sqrt{2}}$ the Higgs VEV plus the Higgs boson, ${ }^{2} y_{i j}$ Yukawa matrix, and $\mathcal{O}$ a set of the BSM and SM particles other than the leptons. $\mathcal{L}_{\mathrm{BSM}}\left[L_{2}, E_{2}, \mathcal{O}\right]$ is a BSM interaction term which can be integrated out at the mass scale $\Lambda$ to induce the $g-2$. The form of $(2.3)$ obviously includes eq. (2.2) but it is more generic than eq. (2.2). Our mechanism will work in a class of the BSM models that can reduce to eq. (2.2) up to the field redefinition. ${ }^{3}$ In this basis the Yukawa matrix $y_{i j}$ naturally has a general flavor structure and I assume $y_{i j}=\mathcal{O}(y)$ independent of $i, j$, where $y$ represents a typical size of the Yukawa coupling. This is taken to be

$$
y \ll 1 .
$$

By integrating out the heavy BSM particles in $\mathcal{O}$, the relevant terms in the effective Lagrangian at the scale $\mu \sim \Lambda$ are

$$
\begin{aligned}
\mathcal{L}_{\text {eff }} \sim & \bar{L}_{1} \partial_{\mu} \gamma^{\mu} L_{1}+Z_{L} \bar{L}_{2} \partial_{\mu} \gamma^{\mu} L_{2}+\bar{e}_{1} \partial_{\mu} \gamma^{\mu} e_{1}+Z_{R} \bar{e}_{2} \partial_{\mu} \gamma^{\mu} e_{2} \\
& -y_{i j} H_{0} L_{i} e_{j}-\delta Y H_{0} L_{2} e_{2} \\
& -\frac{H_{0}}{\Lambda^{2}}\left(\Re\left[C_{\gamma}\right] L_{2} \sigma_{\mu \nu} F_{\mu \nu} e_{2}+i \Im\left[C_{\gamma}\right] L_{2} \gamma_{5} \sigma_{\mu \nu} F_{\mu \nu} e_{2}\right)
\end{aligned}
$$

where $Z_{L, R}$ are the wave function renormalization, $\delta Y$ radiatively generated Yukawa terms, and $C_{\gamma} / \Lambda^{2}$ higher dimensional coupling in chiral representation. Here $\sigma_{\mu \nu} \equiv i\left[\gamma_{\mu}, \gamma_{\nu}\right] / 2$,

\footnotetext{
${ }^{2}$ It is straightforward to add $\mathrm{SU}(2)$ partners for the gauge invariance.

${ }^{3}$ Strictly speaking, there are two classes of renormalizable models leading to the 1loop induced $g-2$ : the loop is composed by only BSM particles, and the loop by BSM and SM particles. eq. (2.1) is a generic Lagrangian of the former case with a single flavor coupling. On the other hand, eq. (2.3) also includes some of the latter case (See the extension to the B-physics anomaly in the last section.)
} 
and $F^{\mu \nu}$ is the field strength of photon. We have neglected the loop effects of order $y^{2}$. The weak boson loops and Higgs wave function renormalization are also neglected since the effects are either chirality suppressed or flavor blind.

Significant corrections are all for the lepton with the index " 2 ". In particular the Yukawa matrix in the effective theory by assuming

$$
|\delta Y| \gg\left|y_{22}\right|
$$

and neglecting $y_{22}$ is

$$
Y_{i j}^{\mathrm{eff}} \propto\left(\begin{array}{cc}
y_{11} & y_{12} / \sqrt{Z_{R}} \\
y_{21} / \sqrt{Z_{L}} & \delta Y / \sqrt{Z_{L} Z_{R}}
\end{array}\right)
$$

with normalized kinetic terms. Here we can take $y_{11}, \delta Y$, to be real positive parameters by performing a field redefinition via chiral rotations of the lepton fields. In this case $y_{12} y_{21}$ is in general a complex parameter. We can easily diagonalize this matrix to get the mass eigenstates of the muon and electron as

$$
L_{\alpha}=U_{L} L, \text { and } e_{\beta}=U_{R} R
$$

where $U_{L, R}$ are unitary matrices satisfying

$$
\begin{aligned}
&\left(U_{L}\right)_{\alpha i} \approx\left(\begin{array}{cc}
1 & -\sqrt{Z_{R}} \frac{y_{12}^{*}}{d Y} \\
\sqrt{Z_{R}} \frac{y_{12}}{d Y} & 1
\end{array}\right) \\
&\left(U_{R}\right)_{\alpha i} \approx\left(\begin{array}{cc}
1 & -\sqrt{Z_{L}} \frac{y_{21}^{*}}{d Y} \\
\sqrt{Z_{L}} \frac{y_{21}}{d Y} & 1
\end{array}\right) .
\end{aligned}
$$

$\alpha=e, \mu$ represents the Yukawa/mass eigenstate. The Eigen masses are found to be

$$
m_{e}=y_{11} v+\mathcal{O}\left(y^{2} / \delta Y\right), \text { and } m_{\mu}=\frac{\delta Y v}{\sqrt{Z_{L}} \sqrt{Z_{R}}}+\mathcal{O}\left(y^{2} / \delta Y\right)
$$

Here we call the heavier lepton the muon and lighter lepton the electron as usual (in the effective theory by integrating out the tau lepton). We can see that eq. (2.8) is the condition that the muon mass is radiatively generated. The effective Lagrangian of the SM has additional terms ${ }^{4}$

$$
\mathcal{L}_{\text {eff }}=-\frac{v}{\Lambda^{2}}\left(\Re\left[\hat{C}_{\gamma}\right]_{\alpha \beta} L^{\alpha} \sigma_{\mu \nu} F_{\mu \nu} e^{\beta}+i \Im\left[\hat{C}_{\gamma}\right]_{\alpha \beta} L^{\alpha} \gamma_{5} \sigma_{\mu \nu} F_{\mu \nu} e^{\beta}\right),
$$

in the EW breaking phase. The coefficients satisfy

$$
\begin{aligned}
\sqrt{Z_{L} Z_{R}} \times \frac{\hat{C}_{\gamma}}{C_{\gamma}} & \approx\left(\begin{array}{cc}
\frac{y_{21} y_{12} \sqrt{Z_{L} Z_{R}}}{\delta Y^{2}} & -\frac{y_{12} \sqrt{Z_{R}}}{\delta Y} \\
-\frac{y_{21} \sqrt{Z_{L}}}{\delta Y} & 1
\end{array}\right) \\
& =\left(\begin{array}{cc}
\frac{y_{12} y_{21}}{y_{11}^{2}} \frac{m_{e}^{2}}{\sqrt{Z_{R} Z_{L}} m_{\mu}^{2}} & -\frac{y_{12}}{y_{11}} \frac{m_{e}}{m_{\mu} \sqrt{Z_{L}}} \\
-\frac{y_{21}}{y_{11}} \frac{m_{e}}{m_{\mu} \sqrt{Z_{R}}} & 1
\end{array}\right) .
\end{aligned}
$$

\footnotetext{
${ }^{4}$ In general we may also have terms of $\bar{L} e \bar{e} e$ contributing to $\mu \rightarrow e e e$. In our scenario, this process is highly suppressed.
} 
$\Re\left[\hat{C}_{\gamma}\right]_{\mu \mu}$ is nothing but the coupling for the muon $g-2$ and $[22]$

$$
\frac{\Re\left[C_{\gamma}\right]_{\mu \mu}}{\Lambda^{2}}=1.03 \times 10^{-5} \mathrm{TeV}^{-2}\left(\frac{\Delta a_{\mu}}{2.51 \times 10^{-9}}\right) .
$$

On the other hand, the $\mu \rightarrow e \gamma$ and electron EDM set bounds to the other components [22]

$$
\frac{\sqrt{\left|\left(C_{\gamma}\right)_{e \mu}\right|^{2}+\left|\left(C_{\gamma}\right)_{\mu e}\right|^{2}}}{\Lambda^{2}} \lesssim 2.1 \times 10^{-10} \mathrm{TeV}^{-2} \sqrt{\frac{\mathrm{Br}_{\mu \rightarrow e \gamma}^{\text {bound }}}{4.8 \times 10^{-13}}}
$$

and

$$
\frac{\Im\left[C_{\gamma}\right]_{e e}}{\Lambda^{2}} \lesssim 5.3 \times 10^{-12} \frac{\left|d_{e}\right|^{\text {bound }}}{1.1 \times 10^{-29} \mathrm{ecm}},
$$

respectively. Here we have used the bounds obtained by the MEG collaboration [23] and the ACME collaboration [24] for the $\mu \rightarrow e \gamma$ and electron EDM, respectively. We arrive at

$$
\sqrt{\frac{\left|y_{12}\right|^{2}}{y_{11}^{2} Z_{L}}+\frac{\left|y_{21}\right|^{2}}{y_{11}^{2} Z_{R}}} \frac{\left|C_{\gamma}\right|}{\Re C_{\gamma}}<0.004\left(\frac{2.7 \times 10^{-9}}{\Delta a_{\mu}}\right) \sqrt{\frac{\mathrm{Br}_{\mu \rightarrow \mathrm{e} \gamma}^{\text {bound }}}{4.8 \times 10^{-13}}}
$$

and

$$
\frac{\Im\left[C_{\gamma} \frac{y_{12} y_{21}}{y_{11}^{2}}\right]}{\Re\left[C_{\gamma}\right]} \frac{1}{\sqrt{Z_{L} Z_{R}}} \lesssim 0.02\left(\frac{\left|d_{e}\right|^{\text {bound }}}{1.1 \times 10^{-29} \mathrm{ecm}}\right)\left(\frac{\Delta a_{\mu}}{2.51 \times 10^{-9}}\right) .
$$

To our model, $\mu \rightarrow e \gamma$ constraint is most restrictive. A necessary condition to satisfy the constraint is

$$
\max \left[\frac{\left|y_{12}\right|}{y_{11} \sqrt{Z_{L}}}, \frac{\left|y_{21}\right|}{y_{11} \sqrt{Z_{R}}}\right] \lesssim 0.001-0.01
$$

This requires a large $\sqrt{Z_{R}}, \sqrt{Z_{L}}$ or/and mild tunings on the off-diagonal components of $y_{i j}$ compared to the diagonal ones. This tuning may be related with UV physics. The suppression may also due to a large value of $\sqrt{Z_{R}}$ and $\sqrt{Z_{L}}$ if the BSM model is strongly coupled, e.g. in a conformal UV theory. We will discuss both cases based on concrete models in the next section and the appendix. In figure 1, we summarize the idea of the mechanism proposed in this paper. In the left panel the tree-level mass vectors of the lepton are shown. Neither of the tree-level mass vectors is aligned with the vector of the dimension 6 photon interaction (along y-axis), which is induced by the BSM loops. By including the radiative induced mass (the right panel), the heavier lepton mass vector, i.e. the muon, is aligned with the $g-2$ direction (=y-axis) The orthogonal vector to the muon mass direction $(\approx$ the $\mathrm{y}$-axis), is the electron. As a consequence the electron EDM and $\mu \rightarrow e \gamma$ rate are highly suppressed.

In perturbation theory $\sqrt{Z_{L, R}}$ can be also large due to the RG running over a large hierarchy, $\Lambda_{\text {cutoff }} \gg \Lambda$. One obtain

$$
Z_{L, R}=\exp \left[\int_{\Lambda_{\text {cutoff }}}^{\Lambda} d \ln \mu \times \gamma_{L, R}\right]
$$

where $\gamma_{L, R}$ are the anomalous dimension of the left handed and right-handed lepton of the flavor "2". Defining $\bar{\gamma}_{L, R} \equiv\left(\int_{\Lambda_{\text {cutoff }}}^{\Lambda} d \ln \mu \times \gamma_{L, R}\right) / \log \left(\Lambda_{\text {cutoff }} / \Lambda\right)$, this is

$$
\sqrt{Z_{L, R}} \sim 400^{\frac{\log \left(\Lambda_{\text {cutoff }} / \Lambda\right)}{30}} \cdot \frac{\bar{\gamma}_{L, R}}{0.4}
$$


The anomalous dimension is a loop suppressed quantity, and $\bar{\gamma}_{L, R}=\mathcal{O}(0.1)$ requires a relatively strong coupling constant of $\mathcal{O}(1)$ in the UV theory. As we will see in the next section, a model can still satisfy the perturbative unitarity.

Since both the lepton flavor violation and CP-violation processes exist, this scenario may be probed by them even if the mass scales of the BSM particles are high. A model independent reach will be the regime with $\max \left[\frac{\left|y_{12}\right|}{y_{11} \sqrt{Z_{L}}}, \frac{\left|y_{21}\right|}{y_{11} \sqrt{Z_{R}}}\right]>10^{-4}-10^{-3}$ since the reach of the $\mu \rightarrow e \gamma$ is $\mathrm{Br}_{\mu \rightarrow e \gamma}^{\text {bound }} \sim 10^{-15}$ at MEG-II [25]. Given an $\mathcal{O}(1)$ CP phase of $C_{\gamma}$, the $\mu \rightarrow e \gamma$ rate and electron EDM have correlation. The both detections will be a smoking gun signature of our scenario. Although it is absent for the model in the next section, our scenario allows the muon to have a large EDM which can be tested in the future by e.g. J-PARC [26-28]. Furthermore, all the $g-2$ scenarios can be fully tested in muon colliders [29-32] even if the heavy particles are beyond the muon collider's reach [31, 32]. In case the BSM particles are within the reach, a particular model may be confirmed by measuring the relevant cross-sections and decay channels of the BSM particles [31].

\section{Lepton-slepton-bino-like system}

To be more concrete, we consider a simplified model similar to the lepton-slepton-bino system in the MSSM models (See refs. [33-37] for the muon $g-2$ explanations, and the both explanations of the muon and electron $g-2[20]$ in this system). The purpose of this section is to study how large the radiatively induced lepton masses and $Z_{L}, Z_{R}$ could be in a perturbative model. We will also discuss extra-dimensional theory embedding the model with mild suppression of $y_{12,21} / y_{11}$ in the appendix.

The Lagrangian is given by

$$
\mathcal{L}_{\mathrm{BSM}}=-g_{L} \bar{L}_{2} \hat{P}_{L} \tilde{L}_{a} \lambda^{a}-g_{R} \bar{e}_{2} \hat{P}_{R} \tilde{e}_{a} \lambda^{a}-A H_{0} \tilde{L}_{a}^{*} \tilde{e}^{a}-\frac{M_{\lambda}}{2} \bar{\lambda}_{a} \lambda^{a}+\text { h.c.. }
$$

Here I define the Lagrangian with normalized kinetic terms at around the renormalization scale $\mu \sim \Lambda ; \lambda_{a}$ are $N$ copies of majorana fermion, "bino"; $\tilde{L}_{a}$, and $\tilde{e}_{a}$ are $N$ copies of "sleptons" of left-handed type and right-handed type, respectively; $g_{L, R}, A, M_{\lambda}$ are couplings and mass parameters. In addition, $m_{L, R}$ will be used for the mass of $\tilde{L}$ and $\tilde{e}$, respectively. The multiplicity of $N$ are introduced for later convenience. Each of $\tilde{L}, \tilde{e}, \lambda$ is a $N$ multiplet of an unbroken $Z_{N}$ symmetry or of $\mathrm{SU}(M)$ gauge symmetry with $M^{2}-1=N$, i.e. $N$ is the adjoint representation (which is the only choice for canceling the gauge anomaly). Thus those particles can only couple to a single linear combination of the leptons. This Lagrangian is more explicitly obtained from eq. (2.1) (plus the Higgs-slepton-slepton interaction) by taking $O^{\mathrm{BSM}, \mathrm{L}}=\tilde{L}_{a} \lambda^{a}, O^{\mathrm{BSM}, \mathrm{R}}=\tilde{e}_{a} \lambda^{a}$, where from the symmetry of $Z_{N}$ or $\mathrm{SU}(M)$ we cannot have the flavor index of $O^{\mathrm{BSM}, \mathrm{L} / \mathrm{R}}$. The redefined Lagrangian (2.2) corresponds to eq. (3.1). We further perform a field redefinition to obtain positive $M_{\lambda}$ and $A$. In this basis, $g_{L} g_{R}^{*}$ has a non-vanishing $\mathrm{CP}$ phase in general.

By integrating out the BSM fields we obtain a radiatively induced Yukawa coupling at the one-loop level:

$$
\frac{\delta Y}{\sqrt{Z_{L} Z_{R}}}=A N \frac{g_{L} g_{R}^{*} M_{\lambda}}{16 \pi^{2}} I\left(M_{1}^{2}, m_{L}^{2}, m_{R}^{2}\right)
$$


Here the loop function is defined as $I(x, y, z)=-\frac{x y \ln (x / y)+y z \ln (y / z)+z x \ln (z / x)}{(x-y)(y-z)(z-x)}$, which satisfies $I(x, x, x)=1 / 2 x$ and $I[x, x, 0] \rightarrow 1 / x$.

Assuming $M_{\lambda} \sim m_{L} \sim m_{R} \sim A / \epsilon \sim \Lambda$ for simplicity, here and hereafter, we obtain

$$
m_{\mu} \sim \frac{|\delta Y|}{\sqrt{Z_{L} Z_{R}}} v \sim 0.1 \mathrm{GeV}\left(\frac{\epsilon N\left|g_{L} g_{R}^{*}\right|}{0.1}\right) .
$$

We note that $\epsilon<1$ is needed for the vacuum stability of the "slepton" potential. In this case, it is easy to generate the lepton mass around $0.1-10 \mathrm{GeV}$. On the other hand, the higher dimensional photon coupling is estimated as (cf. [31])

$$
\frac{C_{\gamma}}{\sqrt{Z_{L} Z_{R}} \Lambda^{2}} \sim \frac{1}{(300 \mathrm{TeV})^{2}}\left(\frac{\epsilon N g_{L} g_{R}^{*}}{0.1}\right)\left(\frac{10 \mathrm{TeV}}{\Lambda}\right) .
$$

In the effective theory, we can remove the CP phases of both $\delta Y$ and $C_{\gamma}$ simultaneously by the field redefinition [18]. However, this is a specific feature of this model and is not satisfied in general UV models for our alignment mechanism. In such a UV model that there is the CP phase in $C_{\gamma}$, the muon EDM is predicted. In the following, we will take $g_{L}, g_{R}$ to be real positives to ease the analysis. Note that we still have the non-vanishing electron EDM from the phase of $y_{12} y_{21}$. From eq. (2.17), surprisingly, the $g-2$ can be explained when the heavy physics mass scale to be even above $\mathcal{O}(10) \mathrm{TeV}$. This is the consequence of the absence of the chirality suppression, i.e. (3.4) is not suppressed by small $y_{i j}$. As we argued in the introduction, the radiative correction to the Yukawa coupling is neither suppressed by the small coupling and a Yukawa coupling much larger than $y_{i j}$ is radiatively induced by the same loop.

As shown in the appendix the $\sqrt{Z_{L, R}}$ can be as large as $\mathcal{O}(10-100)$ for $N=10-10^{3}$ even if the other couplings than $g_{L}$ and $g_{R}$ are negligible (and may be further suppressed by UV dynamics). Therefore by including the full quantum effect, the simple model can be flavor and CP safe if $\left|y_{12,21} / y_{11}\right|<\mathcal{O}(0.1)$. In the case of the introduction of the $\mathrm{SU}(M)$ gauge group or when the SM gauge coupling become strong, the RG running is modified $^{5}$ At the one-loop level, on the other hand, the form of $\gamma_{L, R}$ does not change. Since the gauge contributions slow down the runnings of $g_{L}, g_{R}$, we can estimate the maximized $\sqrt{Z_{L}}$ by taking $g_{L, R}=\sqrt{4 \pi}$ at any scale. Then we obtain

$$
\bar{\gamma}_{L, R}^{\max }=0.24 N
$$

in perturbation theory. In this case from $(2.24)$ we can have $\sqrt{Z_{L, R}} \gg 10-100$, which shows that quantum effect relevant to the $g-2$ can fully solve the flavor and CP problems.

Before ending this section, let me mention that the symmetry, for the multiplicity $N$, stabilizes the lightest BSM particles. In particular $\lambda$ should be a good candidate for dark matter. If $\Lambda$ is not very large, the dark matter can annihilate into the heavy leptons in

\footnotetext{
${ }^{5}$ In particular, we need to consider the effect when $\mathrm{SU}(M)$ is the $\mathrm{SM}$ gauge group, such as the color group $\mathrm{SU}(3)_{c}$. In this case $\lambda_{a}$ could be identified as the PQ fermion whose mass is from the PQ symmetry breaking. As a result, the strong CP problem can be solved by the QCD axion. The axion may be even heavier than the conventional one if $N$ is large and sleptons are lighter than the binos (see the model in the appendix of [38]).
} 
the early and present universe. This is because the lepton coupled to the dark matter automatically becomes the heavy lepton due to the dark matter loop. Thus, we may have the WIMP miracle, and the signal of heavy lepton pair in the indirect detection experiment may be a good probe of this scenario.

\section{Discussion and conclusions}

Radiative tau and electron mass. So far we have focused on the simplified two flavor model. It is straightforward to extend to the three flavor cases. We may introduce another "family" of the BSM particles, e.g. $\tilde{e}_{3}$ and $\tilde{L}_{3}$ in the concrete model, to generate the tau lepton mass. It can be shown to be viable in eq. (3.3) (see also the appendix) that it is easy to generate an $\mathcal{O}(1) \mathrm{GeV}$ fermion mass, especially when the typical mass scale, $\Lambda^{\prime}$, of the sleptons are closer to $\Lambda_{\text {cutoff }}{ }^{6}$ To discuss the flavor and CP violations we can first take the Yukawa matrix $y_{i j}=\mathcal{O}\left(m_{\mu} / v\right)$ for the $i, j=2$ or 3 otherwise $\mathcal{O}\left(m_{e} / v\right)$ with $L_{3}, e_{3}$ being the leptons coupled to the BSM particles of $\tilde{e}_{3}, \tilde{L}_{3}$. This is satisfied in the effective theory that we have discussed by integrating out the family of $\tilde{L}, \tilde{e}$ for muon. By noting that the photon coupling scales with $\Lambda^{\prime}$, but the induced Yukawa coupling does not, one can easily evade the bound for $\tau$ lepton with large $\Lambda^{\prime}$ when we integrate out the $\tilde{e}_{3}$ and $\tilde{L}_{3}$ to generate the tau mass. Therefore the tau mass can be also radiatively induced without spoiling our mechanism. On the other hand, if $\Lambda^{\prime} \sim \Lambda$, we get the $g-2$ of the tau lepton, $\left|\Delta a_{\tau}\right| \sim\left(m_{\tau} / m_{\mu}\right)^{2} \times \Delta a_{\mu} \sim 10^{-7}$. Perhaps, the tau $g-2$ is difficult to be tested in the near future e.g. [39]. The rate of $\tau \rightarrow \mu \gamma$ is around the current bound and this may be tested e.g. in Belle-II experiment [40].

As we can expect, the electron mass can be also induced radiatively by further introducing a weakly coupled family of the BSM particles consistent with the alignment mechanism. In this radiative generation of the Yukawa couplings, the hierarchy of $\left(g_{L} \cdot g_{R}^{*}\right)_{\alpha}$ becomes the hierarchy of the SM Yukawa couplings. In terms of $g_{L} \sim g_{R}$ the hierarchy becomes milder $\sqrt{m_{e}}: \sqrt{m_{\mu}}: \sqrt{m_{\tau}}$. The EDM or $g-2$ of the electron induced by the BSM generating the electron mass can be suppressed if the mass scale is high enough.

Suppression of $\boldsymbol{y}_{\boldsymbol{i j}}$. So far we have assumed the Yukawa coupling in the BSM model satisfying $y \sim m_{e} / v \ll 1$ which is small. However, the small electron mass may be chosen by an anthropic selection [41]. Therefore given that $y_{i j} \sim y, y_{i j}$ may need to be all small due to an anthropic reason.

Alternatively, volume suppression of more complicated manifolds than $S_{1} / Z_{2}$ in extradimension may also explain the small $y_{11,22}$ and even smaller $y_{12,21}$.

Application to B-physics anomaly. One can also apply the mechanism to scenarios explaining other anomalies, like the recently reported B-physics anomaly suggesting a lepton non-universality [17]. Again the BSM generate such non-universality should not induce too large $\mu \rightarrow e \gamma$ rate and the electron EDM. Thus the result also implies the flavor/CP structure of the BSM.

\footnotetext{
${ }^{6}$ We note here that the radiative generation of the tau mass is difficult in the MSSM due to the severe vacuum stability bound for the stau cf. ref. [20].
} 
For instance, the lepton non-universality (and also the muon $g-2$ ) can be explained by a leptoquark e.g. [42, 43] with Lagrangian

$$
\mathcal{L}=\left(\bar{Q}^{c} \lambda_{L} i \tau_{2} L+\bar{u}^{c} \lambda_{R} e\right) \Phi
$$

where $\lambda_{L}$, and $\lambda_{R}$ are Yukawa matrices, and $\Phi$ is the leptoquark with charge $\overline{3}, 1$, and $1 / 3$ under the representation of $\mathrm{SU}(3)_{c}, \mathrm{SU}(2)_{L}$, and $\mathrm{U}(1)_{Y}$. This is nothing but the Lagrangian (3.1) with the replacement $\tilde{L}, \tilde{e}, \lambda \rightarrow \bar{Q}^{c}, \bar{u}^{c}, \Phi$. Therefore with a general flavor structure, the model should be constrained as well by the electron EDM and $\mu \rightarrow e \gamma$ induced by quark-leptoquark loop. Here we propose that if the heavy lepton masses are dominantly generated through the quark-leptoquark loop proportional to top-quark Yukawa coupling, the higher dimensional photon couplings are aligned with the heavy lepton mass vectors. With only top coupling the system can be reduced to (2.3). As a consequence, the lepton flavor and $\mathrm{CP}$ violations relevant to the electron are suppressed. However, if several flavors of quarks are in eq. (4.1), the loops involving lighter quark are in general not aligned to the loop induced by the top quark in the lepton flavor space. Thus there should be suppressed but non-negligible flavor violation. We may need a more or less special quark flavor structure in $\lambda_{L}$ and $\lambda_{R}$. On the other hand, the quark masses are also induced with lepton loops, but it is suppressed by $y_{22} \sim m_{e} / v$ and is negligible.

Conclusions. The large muon $g-2$ but suppressed $\mu \rightarrow e \gamma$ and electron EDM is a hint of the flavor and CP structure of the BSM. Here I studied that the $g-2$ explanation associated with the generation of masses of the heavy SM leptons. I showed that in a large class of models, the $\mu \rightarrow e \gamma$ rate and electron EDM are naturally suppressed since the basis of the higher dimensional operators and masses are automatically aligned. However, they are non-vanishing and can give experimental signals. The further measurement of the $g-2$ together with flavor and $\mathrm{CP}$ violation may reveal the origin of the heavy lepton masses.

Note added: while completing the first version of this paper, I found ref. [44], in which the authors classify the minimal models for both generating a one-loop radiative mass of the muon and the $g-2$. On the contrary, we show that the lepton flavor violation and electron EDM are generically suppressed when the $g-2$ operator and muon mass are radiatively induced at the same time.

\section{Acknowledgments}

The author thanks Tomohiro Abe and Fuminobu Takahashi for useful discussions. The author is also grateful to the University of Tokyo High Energy Physics Theory Group for many bits of help during the COVID epoch when he was a member of the group. This work was supported by JSPS KAKENHI Grant-in-Aid for Scientific Research 19H05810 and $20 \mathrm{H} 05851$.

\section{A RG analysis of the wave function renormalization}

The $\mu \rightarrow e \gamma$ and electron EDM bounds, (2.20) and (2.21), can be satisfied if (2.22) is satisfied. Let us explicitly estimate $Z_{L, R}$. In the renormalization scale $\mu \gtrsim \Lambda$, the anomalous 


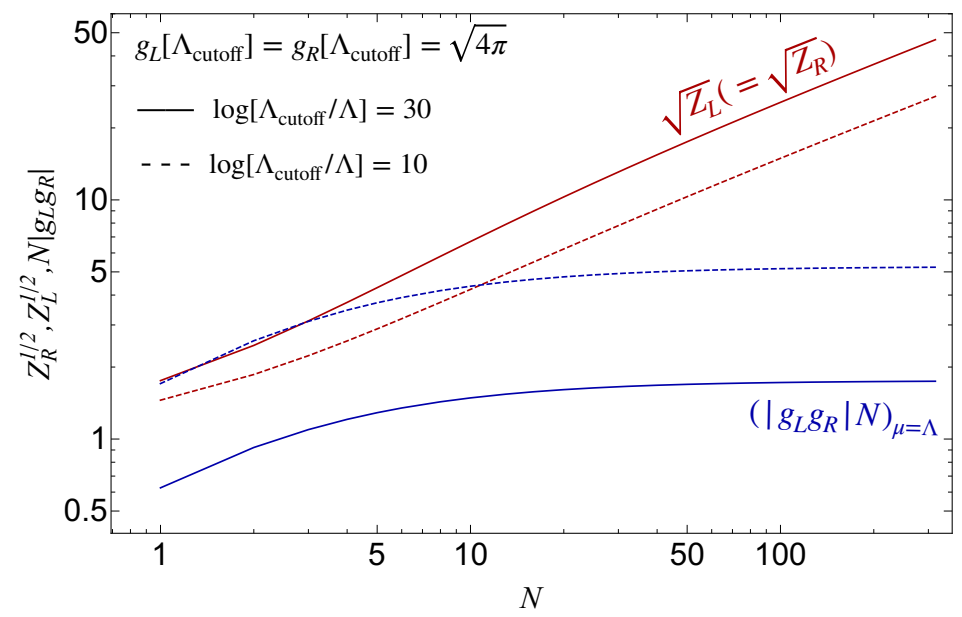

Figure 2. The $\sqrt{Z_{L}}\left(=\sqrt{Z_{R}}\right)$ (red lines) and $\left|g_{L} g_{R}\right| N$ at $\mu=\Lambda$ (blue lines) by varying the multiplicity $N$. The solid (dashed) lines are for the hierarchy $\log \Lambda_{\text {cutoff }} / \Lambda=30$ and 10 respectively.

dimension for $L_{2}\left(e_{2}\right)$ is

$$
\gamma_{L(R)} \sim-\frac{3 N g_{L(R)}^{2}}{16 \pi^{2}}
$$

by neglecting other couplings than $g_{L}, g_{R}$. Also the beta function of $g_{L, R}$ can be obtained as

$$
\begin{aligned}
\frac{d g_{L}}{d \log \mu} \sim \frac{g_{L}}{16 \pi^{2}}\left(\frac{3 N-1}{2} g_{L}^{2}+3 g_{R}^{2}\right), \\
\frac{d g_{R}}{d \log \mu} \sim \frac{g_{R}}{16 \pi^{2}} g_{R} \frac{3 g_{L}^{2}+(3 N+2) g_{R}^{2}}{2}
\end{aligned}
$$

by again neglecting other couplings in this appendix as the minimal scenario. One can solve the RGE to calculate $g_{L}$ and $g_{R}$ and estimate $Z_{L}$ and $Z_{R}$.

To solve the RGE, I take $\Lambda_{\text {cutoff }}$ to be the scale at which $g_{L}=g_{R}=\sqrt{4 \pi}{ }^{7}$ This should give the largest $Z_{L, R}$ for a given $\Lambda_{\text {cutoff }} / \Lambda$. Figure 2 displays $\sqrt{Z_{L}}\left(=\sqrt{Z_{R}}\right)$ with $\Lambda_{\text {cutoff }} / \Lambda \approx 10^{13}$, and $2.2 \times 10^{4}$ for red solid and dashed lines, respectively. We also show the corresponding $N g_{L} g_{R}$ at the renormalization scale $\mu=\Lambda$. This quantity is important for both the induced mass (3.3) and the photon coupling (3.4). One can see that the lepton mass can be as large as a few $\mathrm{GeV}$ with $\Lambda_{\text {cutoff }}$ in an intermediate scale or/and $N$ is large. The $\sqrt{Z_{L, R}}$ can be as large as $\mathcal{O}(10-100)$ for $N=10-10^{3}$ when the other couplings than $g_{L}$ and $g_{R}$ are negligible. Such a large multiplicity may result from an $\mathrm{SU}(M)$ gauge theory. For instance, the adjoint representation of $\mathrm{SU}(5)$ has $N=24$. However, $N \gg 100$ may not be natural, and the 1loop analysis may not be valid. Thus, to evade the $\mu \rightarrow e \gamma$ bound (2.20), we need a mild-tuning of $\max \left[\left|y_{12}\right| / y_{11},\left|y_{21}\right| / y_{11}\right]<\mathcal{O}(10) \%$ unless we take account of other coupling effects like the $\mathrm{SU}(M)$ or SM gauge coupling (see eqs. (2.24) and (3.5)). The $\mathcal{O}(1-10) \%$ tuning region can be tested in the MEG-II experiment.

$y_{12} / y_{11}$ can also be mildly suppressed by UV dynamics. For instance, we can consider that a set of lepton $L_{\text {bulk }}, e_{\text {bulk }}, H_{0}$ live in a bulk in a $5 \mathrm{D}$ model of orbifold $S_{1} / Z_{2}$ at high

\footnotetext{
${ }^{7} g_{L}=g_{R}$ is a (pseudo) IR fixed point, representing an approximate $\mathrm{SO}(3)$ symmetry.
} 
energy. Then from gauge invariance, the $\mathrm{SM}$ gauge bosons (of $\mathrm{SU}(2)_{L} \times \mathrm{U}(1)_{Y}$ ) also live in the bulk. We assume that there is also Yukawa interaction among those particles in the bulk. The other leptons and $\lambda, \tilde{e}, \tilde{L}$ are supposed to live on a brane at the orbifold singularity. This means the slepton-lepton-bino interaction is localized on the brane. Then one finds at the compactification scale, $1 / R$, of the $4 \mathrm{D}$ effective theory that the bulk fields acquire wave functions of $\sim R \times \Lambda_{5}$, with $\Lambda_{5}$ being the $5 \mathrm{D}$ cutoff scale, which we take to be universal for all bulk fields, $R$ the volume of the extra dimension, which is related to

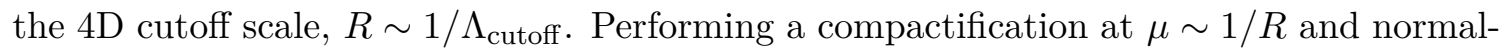
izing the kinetic term, we find that the brane leptons are $\sim L_{2}, e_{2}$ and the bulk leptons are $L_{\text {bulk }} \sim L_{1}, e_{\text {bulk }} \sim e_{1}$ since the bino-bulk [brane] lepton-bulk slepton interaction is suppressed by $\left(R \Lambda_{5}\right)^{-1 / 2}\left[\left(R \Lambda_{5}\right)^{0}\right]$. One also gets $y_{12}, y_{21} \propto\left(R \Lambda_{5}\right)^{-1 / 2}, y_{11}, y_{22} \propto\left(R \Lambda_{5}\right)^{0}$. This means $y_{12} / y_{11}, y_{21} / y_{11}$ is naturally suppressed by $\left(R \Lambda_{5}\right)^{-1 / 2}$. For the $5 \mathrm{D}$ gauge coupling to be weakly coupled at $\mu \sim \Lambda_{5}$, we need $\Lambda_{5} R \lesssim \frac{24 \pi^{2}}{g_{2}^{2}(1 / R)} \sim 10^{3}$. 8 Thus the $\left|y_{12,21} / y_{11}\right| \gtrsim 10^{-2}-10^{-1}$ can be realized in the perturbative regime naturally. This UV model can successfully explain the muon mass as well as the $g-2$ with $N=\mathcal{O}(1)$.

Open Access. This article is distributed under the terms of the Creative Commons Attribution License (CC-BY 4.0), which permits any use, distribution and reproduction in any medium, provided the original author(s) and source are credited.

\section{References}

[1] MuON G-2 collaboration, Final report of the muon E821 anomalous magnetic moment measurement at BNL, Phys. Rev. D 73 (2006) 072003 [hep-ex/0602035] [INSPIRE].

[2] C. Polly, Muon $g-2$ results, seminar at https://theory.fnal.gov/events/event/first-resultsfrom-the-muon-g-2-experiment-at-fermilab/.

[3] Muon G-2 collaboration, Measurement of the positive muon anomalous magnetic moment to 0.46 ppm, Phys. Rev. Lett. 126 (2021) 141801 [arXiv:2104.03281] [inSPIRE].

[4] B.L. Roberts, Status of the Fermilab muon $(g-2)$ experiment, Chin. Phys. C 34 (2010) 741 [arXiv: 1001.2898] [INSPIRE].

[5] M. Davier, A. Hoecker, B. Malaescu and Z. Zhang, Reevaluation of the hadronic vacuum polarisation contributions to the Standard Model predictions of the muon $g-2$ and $\alpha\left(m_{Z}^{2}\right)$ using newest hadronic cross-section data, Eur. Phys. J. C 77 (2017) 827 [arXiv: 1706.09436] [INSPIRE].

[6] A. Keshavarzi, D. Nomura and T. Teubner, Muon $g-2$ and $\alpha\left(M_{Z}^{2}\right)$ : a new data-based analysis, Phys. Rev. D 97 (2018) 114025 [arXiv:1802.02995] [INSPIRE].

[7] A. Keshavarzi, D. Nomura and T. Teubner, $g-2$ of charged leptons, $\alpha\left(M_{Z}^{2}\right)$, and the hyperfine splitting of muonium, Phys. Rev. D 101 (2020) 014029 [arXiv:1911.00367] [INSPIRE].

\footnotetext{
${ }^{8}$ Due to this constraint, one may not explain the $m_{e} / m_{\mu}$ hierarchy purely with the volume suppression. If a certain hierarchy of the Yukawa couplings is set at $\Lambda_{5}$ to explain the SM lepton mass and suppose the BSM fields to explain the $g-2$ without inducing a radiative lepton mass, there are tunings for the alignment between the Yukawa interaction basis and the brane field.
} 
[8] S. Borsányi et al., Leading hadronic contribution to the muon magnetic moment from lattice QCD, Nature 593 (2021) 51 [arXiv:2002.12347] [INSPIRE].

[9] T. Aoyama et al., The anomalous magnetic moment of the muon in the standard model, Phys. Rept. 887 (2020) 1 [arXiv: 2006. 04822] [InSPIRE].

[10] E.-H. Chao, R.J. Hudspith, A. Gérardin, J.R. Green, H.B. Meyer and K. Ottnad, Hadronic light-by-light contribution to $(g-2)_{\mu}$ from lattice QCD: a complete calculation, Eur. Phys. J. C 81 (2021) 651 [arXiv:2104.02632] [INSPIRE].

[11] A. Crivellin, M. Hoferichter, C.A. Manzari and M. Montull, Hadronic vacuum polarization: $(g-2)_{\mu}$ versus global electroweak fits, Phys. Rev. Lett. 125 (2020) 091801 [arXiv: 2003.04886] [INSPIRE].

[12] A. Keshavarzi, W.J. Marciano, M. Passera and A. Sirlin, Muon $g-2$ and $\Delta \alpha$ connection, Phys. Rev. D 102 (2020) 033002 [arXiv:2006.12666] [InSPIRE].

[13] R.H. Parker, C. Yu, W. Zhong, B. Estey and H. Müller, Measurement of the fine-structure constant as a test of the Standard Model, Science 360 (2018) 191 [arXiv:1812.04130] [INSPIRE].

[14] D. Hanneke, S. Fogwell and G. Gabrielse, New measurement of the electron magnetic moment and the fine structure constant, Phys. Rev. Lett. 100 (2008) 120801 [arXiv: 0801.1134] [INSPIRE].

[15] D. Hanneke, S.F. Hoogerheide and G. Gabrielse, Cavity control of a single-electron quantum cyclotron: measuring the electron magnetic moment, Phys. Rev. A 83 (2011) 052122 [arXiv: 1009.4831] [INSPIRE].

[16] K. Petridis and M. Santimaria, New results on theoretically clean observables in rare B-meson decays from LHCb, LHC seminar, March 23 (2021).

[17] LHCb collaboration, Test of lepton universality in beauty-quark decays, arXiv:2103.11769 [INSPIRE].

[18] F. Borzumati, G.R. Farrar, N. Polonsky and S.D. Thomas, Soft Yukawa couplings in supersymmetric theories, Nucl. Phys. B 555 (1999) 53 [hep-ph/9902443] [INSPIRE].

[19] A. Crivellin, J. Girrbach and U. Nierste, Yukawa coupling and anomalous magnetic moment of the muon: an update for the LHC era, Phys. Rev. D 83 (2011) 055009 [arXiv:1010.4485] [INSPIRE].

[20] M. Endo and W. Yin, Explaining electron and muon $g-2$ anomaly in SUSY without lepton-flavor mixings, JHEP 08 (2019) 122 [arXiv:1906.08768] [INSPIRE].

[21] A.E. Cárcamo Hernández, S. Kovalenko, R. Pasechnik and I. Schmidt, Phenomenology of an extended IDM with loop-generated fermion mass hierarchies, Eur. Phys. J. C 79 (2019) 610 [arXiv:1901.09552] [INSPIRE].

[22] A. Crivellin, S. Najjari and J. Rosiek, Lepton flavor violation in the standard model with general dimension-six operators, JHEP 04 (2014) 167 [arXiv: 1312.0634] [INSPIRE].

[23] MEG collaboration, Search for the lepton flavour violating decay $\mu^{+} \rightarrow \mathrm{e}^{+} \gamma$ with the full dataset of the MEG experiment, Eur. Phys. J. C 76 (2016) 434 [arXiv:1605.05081] [INSPIRE].

[24] ACME collaboration, Improved limit on the electric dipole moment of the electron, Nature 562 (2018) 355 [INSPIRE].

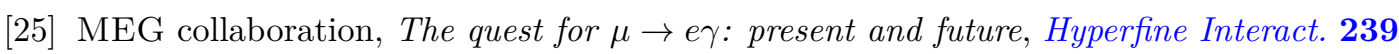
(2018) 58 [arXiv: 1811.05921] [INSPIRE]. 
[26] T.P. Gorringe and D.W. Hertzog, Precision muon physics, Prog. Part. Nucl. Phys. 84 (2015) 73 [arXiv: 1506.01465] [INSPIRE].

[27] A. Crivellin, M. Hoferichter and P. Schmidt-Wellenburg, Combined explanations of $(g-2)_{\mu, e}$ and implications for a large muon EDM, Phys. Rev. D 98 (2018) 113002 [arXiv: 1807.11484] [INSPIRE].

[28] M. Abe et al., A new approach for measuring the muon anomalous magnetic moment and electric dipole moment, PTEP 2019 (2019) 053C02 [arXiv:1901.03047] [INSPIRE].

[29] R. Capdevilla, D. Curtin, Y. Kahn and G. Krnjaic, Discovering the physics of $(g-2)_{\mu}$ at future muon colliders, Phys. Rev. D 103 (2021) 075028 [arXiv:2006.16277] [INSPIRE].

[30] D. Buttazzo and P. Paradisi, Probing the muon $g-2$ anomaly at a muon collider, arXiv: 2012.02769 [INSPIRE].

[31] W. Yin and M. Yamaguchi, Muon g-2 at multi-TeV muon collider, arXiv:2012.03928 [INSPIRE].

[32] R. Capdevilla, D. Curtin, Y. Kahn and G. Krnjaic, A no-lose theorem for discovering the new physics of $(g-2)_{\mu}$ at muon colliders, arXiv:2101.10334 [INSPIRE].

[33] M. Endo, K. Hamaguchi, T. Kitahara and T. Yoshinaga, Probing bino contribution to muon $g-2$, JHEP 11 (2013) 013 [arXiv: 1309.3065] [InSPIRE].

[34] M. Yamaguchi and W. Yin, A novel approach to finely tuned supersymmetric standard models: The case of the non-universal Higgs mass model, PTEP 2018 (2018) $023 \mathrm{~B} 06$ [arXiv: 1606. 04953] [INSPIRE].

[35] W. Yin and N. Yokozaki, Splitting mass spectra and muon $g-2$ in Higgs-anomaly mediation, Phys. Lett. B 762 (2016) 72 [arXiv:1607.05705] [INSPIRE].

[36] T.T. Yanagida, W. Yin and N. Yokozaki, Flavor-safe light squarks in Higgs-anomaly mediation, JHEP 04 (2018) 012 [arXiv:1801.05785] [INSPIRE].

[37] T.T. Yanagida, W. Yin and N. Yokozaki, Muon $g-2$ in Higgs-anomaly mediation, JHEP 06 (2020) 154 [arXiv : 2001.02672] [inSPIRE].

[38] R. Kitano and W. Yin, Strong CP problem and axion dark matter with small instantons, JHEP 07 (2021) 078 [arXiv:2103.08598] [INSPIRE].

[39] L. Beresford and J. Liu, New physics and $\tau$ g-2 using LHC heavy ion collisions, Phys. Rev. D 102 (2020) 113008 [arXiv:1908.05180] [INSPIRE].

[40] BeLLe-II collaboration, The Belle II physics book, PTEP 2019 (2019) 123C01 [Erratum ibid. 2020 (2020) 029201] [arXiv: 1808.10567] [INSPIRE].

[41] V. Agrawal, S.M. Barr, J.F. Donoghue and D. Seckel, Viable range of the mass scale of the standard model, Phys. Rev. D 57 (1998) 5480 [hep-ph/9707380] [INSPIRE].

[42] M. Bauer and M. Neubert, Minimal leptoquark explanation for the $R_{D^{(*)}}, R_{K}$, and $(g-2)_{\mu}$ anomalies, Phys. Rev. Lett. 116 (2016) 141802 [arXiv:1511.01900] [INSPIRE].

[43] E. Coluccio Leskow, G. D'Ambrosio, A. Crivellin and D. Müller, $(g-2)_{\mu}$, lepton flavor violation, and $Z$ decays with leptoquarks: Correlations and future prospects, Phys. Rev. D 95 (2017) 055018 [arXiv: 1612.06858] [INSPIRE].

[44] M.J. Baker, P. Cox and R.R. Volkas, Radiative muon mass models and $(g-2)_{\mu}$, JHEP 05 (2021) 174 [arXiv: 2103.13401] [inSPIRE]. 